\title{
Socio-demographic and injury-related factors contributing to activity limitations and participation restrictions in people with spinal cord injury in Bangladesh
}

\author{
Manzur Kader (i) ${ }^{1} \cdot$ Nirmala K. P. Perera ${ }^{2} \cdot$ Mohammad Sohrab Hossain $^{3,4} \cdot$ Redwanul Islam $^{4}$ \\ Received: 17 February 2017 / Revised: 2 August 2017 / Accepted: 8 August 2017 / Published online: 2 November 2017 \\ (c) International Spinal Cord Society 2018
}

\begin{abstract}
Study design Cross-sectional study.

Objectives To identify socio-demographic and injury-related factors that contribute to activity limitations and participation restrictions in people with spinal cord injury (SCI) in Bangladesh.

Setting Centre for the Rehabilitation of the Paralysed (CRP), Savar, Dhaka, Bangladesh.

Methods This study involved 120 (83\% men) participants with SCI; their median (interquartile range) age and injury duration were 34 (25-43) years and 5 (2-10) years, respectively. Data were collected from the follow-up records kept by the Community Based Rehabilitation (CBR) unit of CRP and a subsequent home visit that included interview-administered questions, questionnaires, and a neurological examination. The dependent variables were activity limitations and participation restrictions, assessed with the World Health Organization Disability Assessment Schedule 2.0 (WHODAS 2.0, scored 0-100; a high score indicates greater activity limitations and participation restrictions). Independent variables included socio-demographic factors (i.e., age, sex, marital status, educational level, monthly household income, employment status, and place of residence) and injury-related factors (i.e., injury duration, cause of injury, injury severity, and type of paralysis). Multivariable linear regression analyses were performed to identify the factors that independently contributed to activity limitations and participation restrictions.

Results Three significant independent variables explained $20.7 \%$ of the variance in activity limitations and participation restrictions (WHODAS 2.0 score), in which tetraplegia was the strongest significant contributing factor, followed by rural residence and complete injury.

Conclusions This study would indicate that tetraplegia, complete injury, and residing in a rural area are the major contributions in limiting the activity and participation following SCI in Bangladesh.
\end{abstract}

Manzur Kader

manzur.kader@med.lu.se

1 Department of Health Sciences, Faculty of Medicine, Lund University, Lund, Sweden

2 Faculty of Health, Federation University Australia, Ballarat, Australia

3 Faculty of Medicine, University of Sydney, Camperdown, Australia

4 Bangladesh Health Professions Institute, Centre for the Rehabilitation of the Paralysed, Savar, Dhaka, Bangladesh

\section{Introduction}

A spinal cord injury (SCI) is a medically complex and lifedisrupting condition which leads to a wide range of functional impairments and health-related problems [1]. It is known that people with SCI experience difficulties with activities of daily living (ADL) and taking part in social activities compared to those without a SCI [2-4]. People living with a SCI in less-resourced countries such as Bangladesh, face challenges due to limited access to health care, rehabilitation programs, and quality-assistive devices [5, 6]. In addition, they are vulnerable to life-threatening complications such as pressure ulcers and urinary tract infections 
after being discharged from a hospital; consequently, many of them die within a few years of the SCI [5]. More specifically, a recent study from Bangladesh reported that $19 \%$ of people who sustained a SCI and are wheelchair-dependent, die within two years of discharge from hospital [7].

There are scarce data on socio-demographic and injuryrelated factors contributing to activity limitations and participation restrictions in people with SCI in less-resourced countries. Previous studies, predominantly from affluent countries, have examined that various factors such as increasing age, time since the injury $[8,9]$, female gender $[9,10]$, and tetraplegia $[8,9,11]$ were found to be associated with activity limitations and participation restrictions in people with SCI. However, it is difficult to generalize these findings to less-resourced regions/countries due to socio-demographic and other contextual differences, such as cultural beliefs, ethnic compositions, and political representation. Previous studies have demonstrated an association between socio-demographic and injury-related characteristics of people with SCI in Bangladesh [6, 12, 13]. For example, a study of 107 patients at the Centre for the Rehabilitation of the Paralyzed (CRP) in 2011 identified that most SCIs (93\%) were caused by traumatic incidents such as a fall from a height, a fall while carrying a heavy load on their head, impact of a heavy object onto the neck and/or back, and due to road traffic accident [13]. Seventy percent of the study participants were less than 40 years old, $83 \%$ of injured people were males, $65 \%$ were married, and $92 \%$ were residents in rural areas. In terms of the characteristics of SCI, tetraplegia and paraplegia accounted for $46 \%$ and $54 \%$ of the injuries, respectively [13]. Another study reported that following SCI, the majority of people were unemployed, house bound, and living in poverty [6].

The aforementioned research from Bangladesh $[6,12,13]$ was limited to the use of univariate or bivariate analyses. Multivariable regression analyses were not used to identify socio-demographic and injury-related factors that independently contribute to activity limitations and participation restrictions in people with a SCI in Bangladesh. The findings will inform clinicians and provide a potential focus for the development of evidence-based interventions and rehabilitation strategies. Accordingly, this study aimed to identify the socio-demographic and injury-related factors that independently contribute to activity limitations and participation restrictions in people with SCI in Bangladesh.

\section{Methods}

This cross-sectional study was conducted on participants with a SCI who were discharged from CRP, living in Savar Upazila (a subdistrict), approximately $25 \mathrm{~km}$ northwest of
Dhaka, the capital of Bangladesh. The CRP is one of the largest SCI units in the world with a recent annual admission of more than 400 patients with SCI across Bangladesh [14]. The patients admitted to CRP include both urban and rural areas.

This study was conducted in accordance with the tenets of the Declaration of Helsinki and received ethical approval (CRP/RE/0401/192) from the CRP. Verbal consent was taken during a phone call prior to each home visit. Written informed consent was sought and gained from all study participants during the home visit.

\section{Participants and recruitment}

The Community Based Rehabilitation (CBR) unit of CRP routinely maintains follow-up records of the patients admitted and discharged from CRP [14]. Records of all the patients were reviewed in April 2015 to identify suitable study participants. A total of 157 patients met the general inclusion criteria: (1) age $>18$ years, (2) they were discharged from CRP after completing comprehensive rehabilitation, (3) were living in the community of Savar in the year 2015, (4) did not possess any other comorbidities (e.g., leg fracture and stroke) along with SCI when discharged and/or whilst living in the community after discharge from CRP, and (5) received at least one follow-up home visit by CRP staff after being discharged. Of 157 participants who were primarily identified for data collection, 17 individuals were unable to contact via the phone, 10 of them declined to participate, five individuals were deceased, three were readmitted to a hospital due to secondary complications along with SCI, and two withdrew their participation. The figure illustrates the recruitment process of the participants in detail.

Consequently, the final sample consisted of 120 participants (83\% male) who were discharged from CRP between June 1981 and February 2014. During this period, 7156 patients were discharged from the CRP. The median (interquartile range, IQR) age was 34 (25-43) years, and the injury duration was $5(2-10)$ years. Table 1 outlines the socio-demographic characteristics of the study participants (Fig. 1).

\section{Data collection}

\section{Procedure}

Data were collected from follow-up records during the recruitment process and a subsequent home visit in person between April 2015 and March 2016, which included interview-administered questions, questionnaires, and a neurological examination. Three trained physiotherapists (one female and two male) conducted the data collection. They were trained regarding questionnaire 
Table 1 Characteristics of the participants, $N=120$

\begin{tabular}{ll}
\hline Variables & Values \\
\hline WHODAS 2.0, mean (SD) & $25(12.8)$ \\
Age, median (IQR) & $34(25-43)$ \\
Sex (men/women), $n(\%)$ & $99(83 \%) / 21(17 \%)$ \\
Marital status (married/unmarried), $n(\%)$ & $74(62 \%) / 46(38 \%)$ \\
Education & \\
Primary or no formal education, $n(\%)$ & $71(59 \%)$ \\
Secondary or above, $n(\%)$ & $49(41 \%)$ \\
Monthly household income & \\
Lower income (<12,000 BDT), $n(\%)$ & $57(47 \%)$ \\
Higher income ( $\geq 12,000$ BDT), $n(\%)$ & $63(53 \%)$ \\
Employment status (employed/unemployed), & $95(79 \%) / 25(21 \%)$ \\
$n \%$ & \\
Place of residence (rural/urban), $n(\%)$ & $78(65 \%) / 42(35 \%)$ \\
Injury duration, median (IQR) & $5(2-10)$ \\
AIS classification, $n(\%)$ & \\
A & $42(35 \%)$ \\
B & $33(28 \%)$ \\
C & $14(12 \%)$ \\
D & $30(25 \%)$ \\
E & $106(88 \%) / 14(12 \%)$ \\
Cause of injury (traumatic/non-traumatic), \\
$n(\%)$
\end{tabular}

AIS (American Spinal Injury Association) ASIA Impairment Scale, $I Q R$ interquartile range, $S D$ standard deviation, WHODAS 2.0 World Health Organization Disability Assessment Schedule 2.0 (0-100, higher $=$ greater activity limitations and participation restrictions

${ }^{\mathrm{a}}$ Complete $=$ AIS A, incomplete $=$ AIS B-E

administration [15] and neurological examination [16] at the CRP prior to data collection.

\section{Variables and instruments}

Dependent variable An interviewer-administered questionnaire using the World Health Organization Disability Assessment Schedule 2.0 (WHODAS 2.0) [15], a generic patient-reported measure was administered to assess activity limitations and participation restrictions of the participants, which constituted the dependent variables of this study. The WHODAS 2.0 is a valid and reliable tool for measuring the disability in people with SCI [17]. By the end of 2015, it has been administered in 94 countries, translated into 47 languages, and dialects including Bengali. It is also used in 27 areas of research $[18,19]$. The WHODAS 2.0 [15] was administered in two previous studies in Bangladesh $[6,20]$. The Bengali version of the WHODAS 2.0 is available from the WHO website: http://www.who.int/classifications/icf/ form_whodas_downloads/en/. Participants were asked to consider how much their disabilities interfered with their lives in the preceding 30 days on a five-point scale ranging from 1 (none) to 5 (extreme/cannot do). It consists of six domains: (1) cognition, (2) mobility, (3) self-care, (4) getting along with others, (5) participation in society, and (6) life activities. The total summary score (the general-disability latent variable) was calculated using the 36-item version simple-scoring template provided on the WHO website: http://www.who.int/classifications/icf/more_whodas/en/. SPSS syntax for recoding and a detailed scoring guideline of WHODAS2.0 has been published by the WHO [15]. All scores ranged from 0 to 100 (a higher score indicates greater activity limitations and participation restrictions).

Independent variables The independent variables (sociodemographic and injury-related data) were selected based on the findings from prior research and their clinical relevance for rehabilitation [6, 8-13]. The socio-demographic data included age (in years), sex (male/female), marital status (married/unmarried), educational level (primary or no formal education/secondary or above), and the total monthly household income which was dichotomized since the data were skewed. Therefore, we categorized this variable into a "low-income group" $(<12,000$ Bangladeshi Taka, BDT) and a "high-income group" ( $\geq 12,000$ BDT) based on the median income value as a cutoff point. The primary employment status was selected based on categories of eight options: (1) paid employment, (2) selfemployment, (3) volunteering, (4) student, (5) homemaker, (6) retired, (7) unemployed due to health, and (8) unemployed due to other reasons. The response options were then merged as "employed (categories 1-4)" and "unemployed" (categories 5-8) [15]. The place of residence was categorized as "urban" or "rural" according to the criteria by Bangladesh Bureau of Statistics [21]. The follow-up records from the CBR unit were cross-checked with medical records to collect specific injury-related data that included the duration of injury (in years), cause of the injury (traumatic/ non-traumatic), severity of the injury (complete/incomplete), and the type of paralysis (tetraplegia/paraplegia). The results of the neurological examination during the home visit were compared to those medical records from CRP. The neurological examination included a sensory examination (light touch and pin prick) and a motor examination on both the right and left side of the body. To determine S4-5 sensory and motor function, voluntary anal contraction and deep anal pressure were tested. The examination guideline, procedure, scoring, and American Spinal Injury Association (ASIA) Impairment Scale (AIS) classification 
Fig. 1 Study participant recruitment process flow diagram

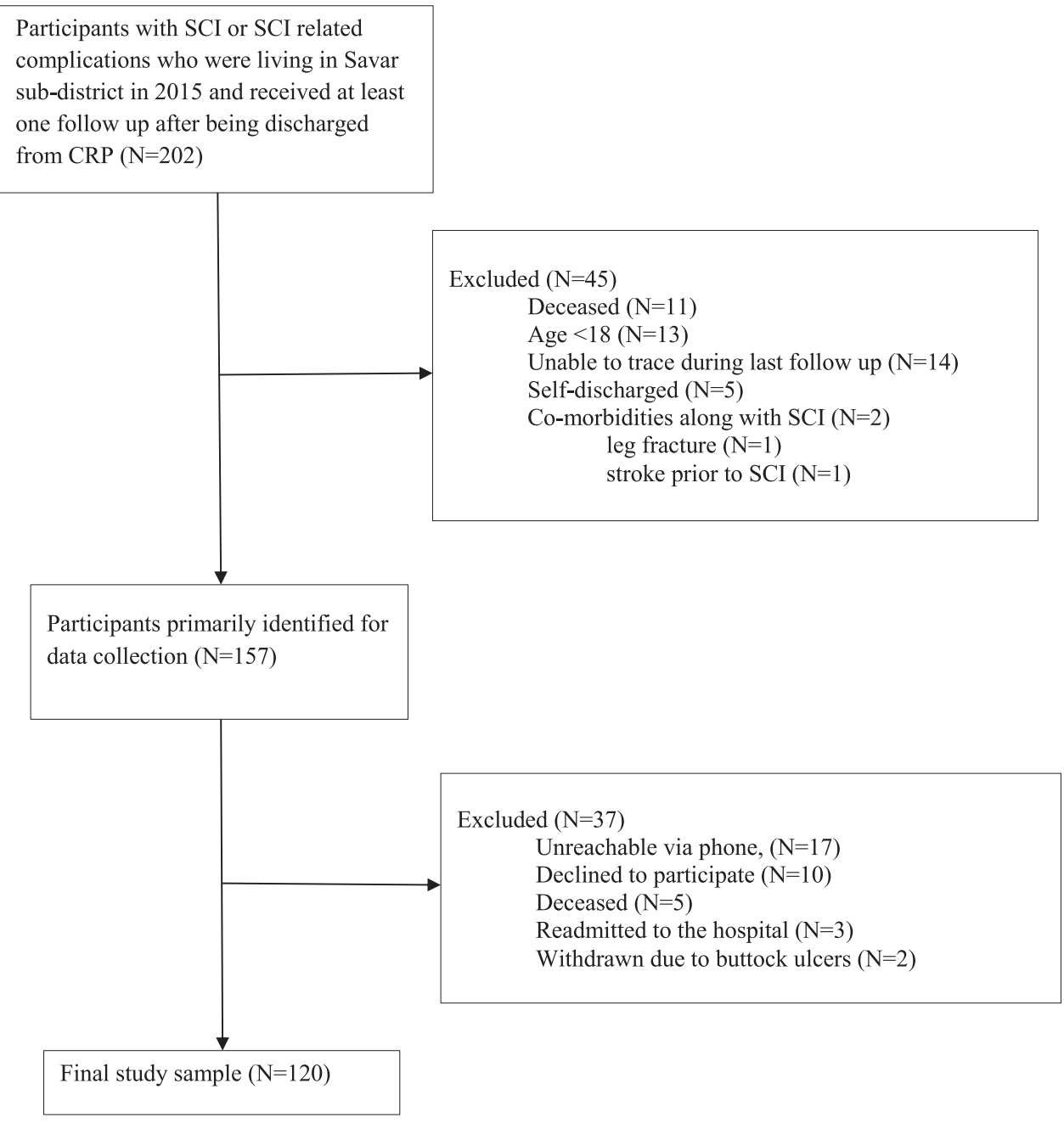

are described in the International Standards for Neurological Classification of Spinal Cord Injury (ISNCSCI) revision 2011 [16]. The manual ISNCSCI worksheet (2013 version) was used to record the data of the neurological examination, which has been developed based on ISNCSCI revision 2011 [22]. It is recognized that reporting errors occur in relation to motor-level determination using the AIS classification and the associated worksheets despite formal training [23]. Therefore, to classify the severity of injury according to AIS grade A-E, raw ISNCSCI worksheet data were entered into a free validated [24], online computerized Rick Hansen Institute algorithm (version 1.0.3) [25]. The algorithm is based on the 2013 revised version of the ISNCSCI worksheet [24]. The type of paralysis was defined in accordance with the ISNCSCI 2011 revision booklet [16], and verified by comparing the neurological examination undertaken by the assessors at the home visit against medical records. For descriptive purposes, mobility status of the participants was categorized as "walking" (walk independently or with an assistive device indoor/outdoor) or "wheelchair-dependent" was assessed during the home visit.

\section{Statistical analysis}

Data were checked regarding the underlying assumptions (i.e., a linear relationship, independent, and normally distributed residuals with constant variance) for linear regression and all the assumptions were satisfied. Descriptive statistics were computed for all the variables. Ordinal and continuous variables are expressed by means (SD) or a median (interquartile range), depending on the distribution of the data (i.e., the normality was checked), and categorical variables are described by the number of patients (percentage). Pearson (r) or Spearman (rs) correlations were used as appropriate to assess the relationships among independent variables to identify any multi-collinearity. There was no sign of multi-collinearity in the independent variables.

Firstly, a simple linear regression analysis was used to investigate the influence of each independent variable on the dependent variable (the WHODAS 2.0). Secondly, all variables with a $P$-value below 0.25 were then entered into a multivariable linear regression model [26] to determine their associations with the dependent variables. Thirdly, eight 
Table 2 Simple linear regression analyses with WHODAS 2.0 score as the dependent variable in people with spinal cord injury, $N=120$

\begin{tabular}{llll}
\hline Independent variables & $\begin{array}{l}\text { Unstandardized coefficient B } \\
(95 \% \text { CI })\end{array}$ & $\begin{array}{l}\text { Standardized coefficient } \\
\beta\end{array}$ & $\begin{array}{l}P \text {-value } \\
\text { Sex (women) }\end{array}$ \\
Age (years) & $-1.61(-7.73,4.52)$ & -0.05 & 0.605 \\
Marital status (unmarried) & $4.26(-0.47,8.99)$ & -0.06 & 0.507 \\
Education (primary or no formal & $1.49(-3.24,6.22)$ & 0.16 & 0.077 \\
education) & & 0.06 & 0.534 \\
Monthly household income (lower, & $3.72(-0.90,8.33)$ & 0.15 & 0.113 \\
$<12,000$ BDT) & & & \\
Employment status (unemployed) & $7.36(1.79,12.94)$ & 0.23 & 0.010 \\
Place of residence (rural) & $5.29(0.51,10.08)$ & 0.20 & 0.030 \\
Injury duration (years) & $-0.50(-0.90,-0.09)$ & -0.22 & 0.017 \\
Cause of injury (traumatic) & $6.81(-0.33,13.96)$ & 0.17 & 0.061 \\
Injury severity (complete) & $5.68(0.90,10.44)$ & 0.21 & 0.020 \\
Type of paralysis (tetraplegia) & $7.10(2.10,12.10)$ & 0.25 & 0.006 \\
\hline Note: WHODAS 2.0 World Health & Organization Disability Assessment Schedule $2.0(0-100$, higher $=$
\end{tabular}

greater activity limitations and participation restrictions) dichotomous or continuous independent variables were entered into a multivariable regression model. The $P$-values for all independent variables in this model were inspected and the variable with the highest $P$-value was manually removed from the model. This step was repeated until all independent variables in the model had $P$-values $<0.1$, which became the final model.

The data were analyzed using the SPSS v. 23 software for Windows (IBM Corporation, Armonk, 137 New York, United States). All $P$-values reported are based on twotailed comparisons where applicable and the statistical level of significance was set at alpha $5 \%(P<0.05) . P$-values were presented exactly except when they were below 0.001 .

\section{Results}

The mean (SD) WHODAS 2.0 score was 25 (12.8). The results from the univariate analyses are presented in Table 2. Of all the independent variables, tetraplegia was the strongest variable $(\beta=0.25, \quad P<0.006)$ and sex (women) was the weakest variable $(\beta=-0.05, P=0.605)$ related to activity limitations and participation restrictions (WHODAS 2.0 score) (Table 2).

Five independent variables emerged in the final model of the multivariable linear regression analysis that could explain $20.7 \%$ of variance in the disability (WHODAS 2.0 score), of which three variables were statistically significant. The strongest independent variable (as assessed by the standardized regression coefficient, $\beta$ ) was tetraplegia $(\beta=0.31, \quad P<0.001)$, followed by rural residence $(\beta=0.21, \quad P=0.017)$ and complete injury $(\beta=0.20$, $P=0.020)($ Table 3$)$.

\section{Discussion}

This is the first cross-sectional study to investigate the association between socio-demographic and injury-related factors and activity limitations and participation restrictions in people with SCI living in Savar Upazila of Bangladesh using a multivariable regression analysis. Tetraplegia, rural residence, and complete injury emerged as important factors that influence activity limitations and participation restrictions in people with SCI.

Due to the lack of other published data for SCI patients in Bangladesh, or countries from the region, comparisons were expanded to include studies from affluent countries. Our findings concur with previous studies from the United States [8, 9] and Sweden [11] where tetraplegia was identified as the strongest factor associated with activity limitations in people with SCI. A possible explanation could be that people with tetraplegia encounter greater physical challenges than those with paraplegia. As tetraplegia affects all four limbs, a person with tetraplegia is more likely to experience more spasticity [27], cardiorespiratory complications [28, 29], and poorer hand function [30] than a person with paraplegia. Consequently, people with tetraplegia might experience more profound difficulties with ADL such as hand-to-mouth activity, reaching, grasping, gripping, and pinching as a result of poor hand function [30].

This study suggests that a complete SCI status is a contributing factor to activity limitations and participation restrictions in people with SCI. It is likely that those with a complete SCI might experience more spasticity [27] and cardiorespiratory complications $[28,29]$ than people with incomplete SCI, thus affecting their physical independence. 
Table 3 Multivariable linear regression analyses with WHODAS 2.0 score as the dependent variable in people with spinal cord injury, $N=120$

\begin{tabular}{llll}
\hline Independent variables $^{\mathrm{a}}$ & $\begin{array}{l}\text { Unstandardized coefficient } \\
\mathrm{B}(95 \% \mathrm{CI})\end{array}$ & $\begin{array}{l}\text { Standardized } \\
\text { coefficient } \beta\end{array}$ & $P$-value $R$ square \\
\hline Type of paralysis (tetraplegia) & $8.69(3.90,13.48)$ & 0.31 & $<0.7 \%$ \\
Place of residence (rural) & $5.60(1.02,10.18)$ & 0.21 & 0.017 \\
Injury severity (complete) & $5.32(0.84,9.80)$ & 0.20 & 0.020 \\
Monthly household income & $3.97(-0.46,8.40)$ & 0.16 & 0.079 \\
(lower, <12,000 BDT) & & & 0.085 \\
Marital status (unmarried) & $3.87(-0.54,8.27)$ & 0.15 & 0.001 \\
\hline
\end{tabular}

Note: WHODAS 2.0 World Health Organization Disability Assessment Schedule 2.0 (0-100, higher = greater activity limitations and participation restrictions)

${ }^{a}$ The following eight independent variables were included: marital status, monthly household income, employment status, place of residence, injury duration, cause of injury, injury severity, and type of paralysis
In addition, these people with a complete SCI might be prone to secondary health conditions such as bowel- and bladder-related problems, and respiratory illness, severe nociceptive, and/or neuropathic pain also influence their activity limitation [11]. Therefore, regardless of the resource availability, ADL and participation will be more limited if a person has a complete SCI. In addition, the type of injury may dictate the resources and the type of interventions required because of varied levels of physical independence. Therefore, people with a complete SCI require improved access ongoing rehabilitation services, affordable mobility aid devices such as the powered wheelchairs, and home care services to live more independently.

This study adds to the body of evidence linking a rural residence to increased activity limitation for people with SCI [31]. The potential reasons for this are complex and are likely to include a limited availability and access to health care and rehabilitation services. In addition, the physical and architectural barriers such as the lack of infrastructure may contribute towards activity limitations and participation restrictions to people with SCI, particularly those who are living in the rural residence.

A strength of the present study is that it renders novel knowledge concerning the factors contributing to activity limitations and participation restrictions in people with SCI, an area where currently there is limited knowledge in lessresourced countries. The sample size allowed us to consider a variety of socioeconomic and disease-related factors for the use of a multivariable regression model. That is, it enabled the investigation of complex relationships with multiple interacting factors providing advantages over bivariate analyses. No prior study in Bangladesh has used multivariable regression analysis to investigate the sociodemographic and injury-related factors that independently contribute to activity limitations and participation restrictions in people with SCI. It is noteworthy that employment status and the duration of injury were not independently associated to activity limitations and participation restrictions when controlling for the other independent variables, despite the highly significant univariate association demonstrated. This finding illustrates a major pitfall in relying on univariate analyses.

The present study has, however, some limitations. Given the complexity of activity limitations and participation restrictions in SCI, it is fair to presume that there are additional factors or secondary complications that remain unexplained in our model. Factors such as spasticity, presence of pressure ulcers, and pain need to be included in the analysis of future studies.

This study included those who were discharged from the CRP, a specialist rehabilitation center for SCI in Bangladesh. The current CBR policy states that when patients are discharged, they will be receiving follow-up home visits. The aim of the home visit is to screen the necessity of readmission, potential therapeutic interventions, and modifications to the home environment to ensure accessibility as per the CBR policy of CRP [14]. It should be noted that it is not known when this CBR service was introduced. This study population includes the discharge of patients since 1981. Therefore, it is difficult to be confident that all patients discharged from 1981 received a similar follow-up home visit. Also, in the absence of a clear outline, it is unclear if patients received a follow-up visit routinely, or the patients were randomly selected by the CBR staff [14]. If a patient did not receive a follow-up visit, then, it is possible that they might not be included in this study sample. Thus, the findings are subject to the inherent limitations associated with this study population. Evaluating the impact of the "follow up" visit on activity and participation over time is an area of interest for future research. In addition, investigating the methods to optimize follow-up visits to achieve improved functional outcomes is an area of interest, particularly in resource-poor settings such as Bangladesh.

Although the data were from the CRP, the largest SCI rehabilitation center in Bangladesh [14], the results from 
this study should be interpreted with caution. This is because the participants included in this study were a sample of convenience and therefore, they were not reflective of patients with SCI discharged from other facilities in Bangladesh that may not have access to the same resources such as a dedicated rehabilitation center, mobility devices, and post discharge follow-up home visits. Moreover, the current study sample might not be representative of all the patients discharged from CRP during the same period and is a limitation of this study. To gain insights into whether this sample reflects those typically discharged from CRP, we compared the current study population against a similar study with a representative sample of all the patients discharged from the CRP in 2011 [7]. Our sample had 83\% male vs. $89 \%$, and the median age was 30 years vs. 32 years. In addition, there were $72 \%$ people with paraplegia and $64 \%$ were wheel-chair dependent in the current study compared to 61 and $65 \%$ of people in the study by Hossain et al. [7]. Therefore, it is likely that the current study is biased with an overrepresentation of people with paraplegia. Moreover, we acknowledge that these participants could have had particular characteristics (e.g., more complications and needs). This limits the generalizability of the findings. Another limitation of this study was recall bias due to the self-reported nature of the data. For example, limitations related to memory, misreporting of exposure, and social desirability [32] may have affected the validity of the data. The following steps were taken to minimize the recall bias. Trained professional interviewed the study participants when necessary clarifications were provided to reduce the bias in the interpretation of the questions. Questionnaire responses were limited to standardized options to increase the reliability of the responses. In addition, there are limitations to the cross-sectional design of this study. For example, this approach does not allow for interpretation about cause-and-effect relationships. More robust methods and longitudinally collected data are required to compare them against the findings of this study.

In conclusion, tetraplegia, a rural residence, and complete injury emerged as important factors associated with activity limitations and participation restrictions in people with SCI in Bangladesh. The results of this study may provide some useful information for the development of evidence-based interventions.. There is a great need to develop interventions based on the severity of injury. Moreover, a rural residence should be in focus while developing interventions to reduce activity limitations and participation restrictions in people with SCI. Since this is the first study from Bangladesh that used multivariable analysis to identify the contributing factors to activity limitations and participation restrictions in people with SCI, the present findings need to be replicated in other populations with SCI in other parts of Bangladesh. Moreover, future research would benefit from the longitudinal design to identify the predictive factors and understand how activity limitations and participation restrictions evolve over time in people with SCI.

\section{Data archiving}

All relevant data are within this manuscript and raw data are archived by the authors.

Acknowledgements We thank the participants for their cooperation, and the physiotherapists Rasel Howlader and Maisha Hoque for data collection. We gratefully acknowledge Kazi Imdadul Hoque, a clinical physiotherapist, CRP for his valuable suggestions to the manuscript.

\section{Compliance with ethical standards}

Conflict of interest The authors declare that they have no competing interests.

\section{References}

1. Kirchberger I, Biering-Sorensen F, Charlifue S, Baumberger M, Campbell R, Kovindha A, et al. Identification of the most common problems in functioning of individuals with spinal cord injury using the International Classification of Functioning, Disability and Health. Spinal Cord. 2010;48:221-9.

2. Kemp B, Adkins R, Thompson L. Aging with a spinal cord injury: what recent research shows. Top Spinal Cord Inj Rehabil. 2004;10:175-197.

3. Thompson L, Yakura J. Aging Related Functional Changes in Persons with Spinal Cord Injury. Top Spinal Cord Inj Rehabil. 2001;6:69-82.

4. Barclay L, McDonald R, Lentin P. Social and community participation following spinal cord injury: a critical review. Int $\mathrm{J}$ Rehabil Res. 2015;38:1-19.

5. World Health Organization. International perspectives on spinal cord injury; 2013.

6. Hossain MS, Rahman MA, Bowden JL, Quadir MM, Herbert RD, Harvey LA. Psychological and socioeconomic status, complications and quality of life in people with spinal cord injuries after discharge from hospital in Bangladesh: a cohort study. Spinal Cord. 2015;54:483-9.

7. Hossain MS, Rahman MA, Herbert RD, Quadir MM, Bowden JL, Harvey LA. Two-year survival following discharge from hospital after spinal cord injury in Bangladesh. Spinal Cord. 2015;54:132-6.

8. Whiteneck G, Meade MA, Dijkers M, Tate DG, Bushnik T, Forchheimer MB. Environmental factors and their role in participation and life satisfaction after spinal cord injury. Arch Phys Med Rehabil. 2004;85:1793-803.

9. Whiteneck G, Tate D, Charlifue S. Predicting community reintegration after spinal cord injury from demographic and injury characteristics. Arch Phys Med Rehabil. 1999;80:1485-91.

10. Krause JS, Broderick L. Outcomes after spinal cord injury: comparisons as a function of gender and race and ethnicity. Arch Phys Med Rehabil. 2004;85:355-62.

11. Jorgensen S, Iwarsson S, Lexell J. Secondary health conditions, activity limitations, and life satisfaction in older adults with longterm spinal cord injury. PM \& R. 2016;9:356-66. 
12. Hoque MF, Grangeon C, Reed K. Spinal cord lesions in Bangladesh: an epidemiological study 1994-1995. Spinal Cord. 1999;37:858-61.

13. Islam MS, Hafez MA, Akter M. Characterization of spinal cord lesion in patients attending a specialized rehabilitation center in Bangladesh. Spinal Cord. 2011;49:783-6.

14. Centre for the Rehabilitation of the Paralyzed. Annual report: 2014-2015, celebrating 36 years of service. Bangladesh: CRP Printing Press; 2015.

15. Ustun T, Kostanjsek N, Chatterji S, Rehm J. Measuring health and disability: manual for WHO disability assessment schedule (WHODAS 2.0). Geneva, Switzerland: World Health Organization; 2010.

16. Kirshblum SC, Burns SP, Biering-Sorensen F, Donovan W, Graves DE, Jha A, et al. International standards for neurological classification of spinal cord injury (revised 2011). J Spinal Cord Med. 2011;34:535-46.

17. Wolf AC, Tate RL, Lannin NA, Middleton J, Lane-Brown A, Cameron ID. The World health organization disability assessment scale, WHODAS II: reliability and validity in the measurement of activity and participation in a spinal cord injury population. J Rehabil Med. 2012;44:747-55.

18. Ustun TB, Chatterji S, Kostanjsek N, Rehm J, Kennedy C, Epping-Jordan J, et al. Developing the World Health Organization disability assessment schedule 2.0. BullWorld Health Organ. 2010;88:815-23.

19. Federici S, Bracalenti M, Meloni F, Luciano JV. World Health Organization disability assessment schedule 2.0: an international systematic review. Disabil Rehabil 2016;39:2347-80.

20. Fitch T, Villanueva G, Quadir MM, Sagiraju HK, Alamgir H. The prevalence and risk factors of post-traumatic stress disorder among workers injured in rana plaza building collapse in Bangladesh. Am J Ind Med. 2015;58:756-63.

21. Population Housing Census. Bangladesh Bureau of Statistics. 2011. http://203.112.218.65/WebTestApplication/userfiles/Image/ National\%20Reports/PopulationHousingCensus2011.pdf. Accessed 16 Feb 2017.
22. Kirshblum S, William W. Updates for the international standards for neurological classification of spinal cord injury. Phys Med Rehabil Clin N Am. 2014;25:505-17.

23 Schuld C, Wiese J, Franz S, Putz C, Stierle I, Smoor I, et al. Effect of formal training in scaling, scoring and classification of the international standards for neurological classification of spinal cord injury. Spinal Cord. 2013;51:282-88.

24 Walden K, Belanger LM, Biering-Sorensen F, Burns SP, Echeverria E, Kirshblum S, et al. Development and validation of a computerized algorithm for international standards for neurological classification of spinal cord injury (ISNCSCI). Spinal Cord. 2016;54:197-03.

25 Rick Hansen Institute. ISNCSCI algorithm. http://www.isncscia lgorithm.com/. Accessed 22 June 2017.

26. Katz MH. Multivariable analysis: a practical guide for clinicians and public health researchers. Cambridge: Cambridge University Press; 2011.

27. Skold C, Levi R, Seiger A. Spasticity after traumatic spinal cord injury: nature, severity, and location. Arch Phys Med Rehabil. 1999;80:1548-57.

28. Ravensbergen HJ, de Groot S, Post MW, Slootman HJ, van der Woude LH, Claydon VE. Cardiovascular function after spinal cord injury: prevalence and progression of dysfunction during inpatient rehabilitation and 5 years following discharge. Neurorehabil Neural Repair. 2014;28:219-29.

29. Jackson AB, Groomes TE. Incidence of respiratory complications following spinal cord injury. Arch Phys Med Rehabil. 1994;75:270-5.

30. Harvey LA, Batty J, Jones R, Crosbie J. Hand function of C6 and C7 tetraplegics 1-16 years following injury. Spinal Cord. 2001;39:37-43.

31. Sekaran P, Vijayakumari F, Hariharan R, Zachariah K, Joseph SE, Kumar RK. Community reintegration of spinal cordinjured patients in rural south India. Spinal Cord. 2010;48: 628-32.

32. Fisher RJ. Social desirability bias and the validity of indirect questioning. J Consumer Res. 1993;20:303-315. 